\title{
Update on late-onset hypogonadism (LOH) or ADAM: treatment
}

\section{AtualizaÇÃo EM hipogonadismo MASCULINo taRdio (HMT) ou DAEM: tratamento}

Bernardo WM, Martits AM, Costa EMF, Nardi AC, Nardozza Jr A, Facio Jr FN, Faria G

1. What is the role of androgen replacement therapy (ART) to restore bone mass, muscular strength and body composition?

a. Increase in fat mass.

b. Does not affect bone mass.

c. Increase in muscle mass.

d. Reduces the testicular volume.

2. What is the role of androgen replacement therapy (ART) to restore libido and sexual function?

a. Testosterone restores libido in men independent of the hormone's levels.

b. Testosterone restores libido in men with low testosterone levels.

c. The combination of testosterone and phosphodiesterase inhibitors produces no benefit.

d. Dehydroepiandrosterone (DHEA) improves erectile dysfunction.

3. What is the risk of ART in relation to exacerbating prostate disease?

a. Increases the incidence of prostate cancer

b. Cannot be used in patients treated for prostate cancer.

c. Patients with a family history of prostate cancer should not use ART.

d. Can increase PSA levels.

4. How should ART be administered orally?

a. $50 \mathrm{mg}$ of DHEA orally twice a day, improves sexual function in men.

b. Oral testosterone undecanoate (OTU),160 mg/day, improves sexual function.

c. Oral mucosa patches (containing $30 \mathrm{mg}$ of testosterone), 3 times a day.

d. It is strongly advised NOT to use oral testosterone formulations.
5. What is the best form of ART?

a. Injectable testosterone undecanoate $\operatorname{depot}(\mathrm{TUD})$ is the most physiologic.

b. The oral route remains a quick and convenient way to ART.

c. Skin patches at the maximum dose are not very effective.

d. Subcutaneous testosterone implants can be used in our midst.

AnsWers to Clinical SCENARIO: ANGLE-CLOSURE GLAUCOMA: TREATMENT [PUBLISHED IN RAMB 2014; 60(5)]

1. On prophylactic laser iridotomy, in the contralateral eye, in a patient with acute primary angle closure in one eye, we can affirm that:

It can prevent similar episodes in many cases. (Alternative $\mathbf{A}$ )

2. The differences between prophylactic laser iridotomy (PLI) and prophylactic surgical iridectomy include:

PLI costs less. (Alternative $\mathbf{B}$ )

3. It is not a complication of PLI:

Clinical improvement of cataract. (Alternative $\mathbf{C}$ )

4. Which anatomic situation of the camerular sinus is an indication for prophylactic laser iridotomy? All cases diagnosed as primary angle closure. (Alternative $\mathbf{B}$ )

5. Factors involved in the indication of PLI in cases of suspected primary angle closure include all of the following, except:

Lack of symptoms. (Alternative A) 


\section{ANSWERS TO CLINICAL SCENARIO: LATE-ONSET HYPOGONADISM (LOH) OR ADAM: DIAGNOSIS [PUBLISHED IN RAMB 2014; 60(5)]}

1. The main signs and symptoms involved in late-onset male hypogonadism include the following, except:

Anxiety. (Alternative B)

2. On the main instruments used in the definition and diagnostic assessment of male aging, we cannot affirm that:

The use of the three instruments makes the diagnosis of HMT through functional and biochemical criteria unnecessary. (Alternative $\mathbf{D}$ )
3. What is the role of the serum and free testosterone (BAT) levels in the diagnosis of late-onset hypogonadism?

TT (total testosterone) would not be the ideal measure to assess late-onset hypogonadism. (Alternative $\mathbf{C}$ )

4. Tests to be requested before the start of hormone replacement therapy (ART) include the following, except:

Evaluation of renal function. (Alternative $\mathbf{D}$ )

5. How should prostate cancer be monitored?

Ultrasound-guided prostate biopsy. (Alternative A) 